\title{
Influence of Surfactants and Dissolved Gases on the Silver Nanoparticle Plasmon Resonance Absorption Spectra Formed by the Laser Ablation Processes
}

\author{
Ming Jing Chua ${ }^{1}$ and Yoshinori Murakami \\ ${ }^{1}$ Department of Materials Science, Nagaoka National College of Technology, Nagaoka, Niigata 940-2188, Japan \\ ${ }^{2}$ Department of General Science, Hachinohe National College of Technology, Hachinohe, Aomori 039-1104, Japan \\ Correspondence should be addressed to Yoshinori Murakami; murakami-g@hachinohe-ct.ac.jp
}

Received 10 April 2013; Accepted 15 May 2013

Academic Editors: D. Knopf, T. Panczyk, and S. Sasaki

Copyright ( $\odot 2013$ M. J. Chua and Y. Murakami. This is an open access article distributed under the Creative Commons Attribution License, which permits unrestricted use, distribution, and reproduction in any medium, provided the original work is properly cited.

\begin{abstract}
The silver nanoparticles were fabricated by the focused laser irradiation to silver rod immersed in various kinds of surfactant aqueous solutions. It was found that anionic and cationic surfactants showed different roles on the silver nanoparticle growth during the focused laser irradiation processes. Silver nanoparticle synthesis in an amphoteric surfactant aqueous solution was also carried out using the same techniques, and it was found that the spectral shifts for these surface plasmon bands showed complicated behaviors against the concentration of amphoteric surfactants as well as $\mathrm{pH}$. Furthermore, the influence of the gas dissolved in a solution on the silver nanoparticle growth during the focused laser irradiation processes was investigated. With increasing the gas pressure of $\mathrm{CO}_{2}$, the surface plasmon bands of silver nanoparticles were shifted to longer wavelength, suggesting that the dissolved gas of $\mathrm{CO}_{2}$ in a solution enhances the silver nanoparticle growth. The plausible mechanism was proposed to understand the reason of such enhancement of silver nanoparticle growth by increasing the dissolved gas in a solution.
\end{abstract}

\section{Introduction}

Nanoscale materials have attracted much attention due to their unique properties being different from their bulk materials [1]. For example, gold and silver nanoparticles have been used in various analytical techniques such as catalysis, biosensing, recording media, and photoscience. From the production point of view, chemical reduction of metal ions is mostly commonly employed in the preparation of metal nanoparticles in solution. In the last few years, a new methodology based on the laser ablation of bulk metal in water appeared for generating metal nanoparticles. The use of surfactants, especially, which covers the particles during their condensation, promotes improved size uniformity as well as reduces the coalescence during the fabrication of nanoparticles using such laser ablation techniques. Mafuné et al. [2] reported for the first time that the size distribution of silver nanoparticles produced by the laser ablation varied with the addition of sodium dodecyl sulfate (denoted by
SDS hereafter) in water, and they also reported that the size became smaller and narrower with increasing the concentration of SDS. The authors also attempted the formation of stable platinum nanoparticles using the laser ablation of platinum plate in water and found that the particle diameter of platinum nanoparticles decreased with increasing the concentration of SDS [3]. Although the effects of the anionic surfactants of SDS on the size distribution of the laser ablated metal nanoparticles have been thoroughly investigated, very few works have been done for the influences on the other anionic and cationic surfactants.

As an example of the investigations on the influence of a cationic surfactants, Chen and Yeh [4] have investigated the particle diameters of silver nanoparticles formed by the laser ablation of silver rod from the enlarged transmission electron photographs and found that the particle diameters were $4.2 \mathrm{~nm}$ and $7.8 \mathrm{~nm}$ with the addition of the anionic SDS and the cationic cetyltrimethylammonium bromide (denoted by CTAB hereafter), respectively. They concluded that the 


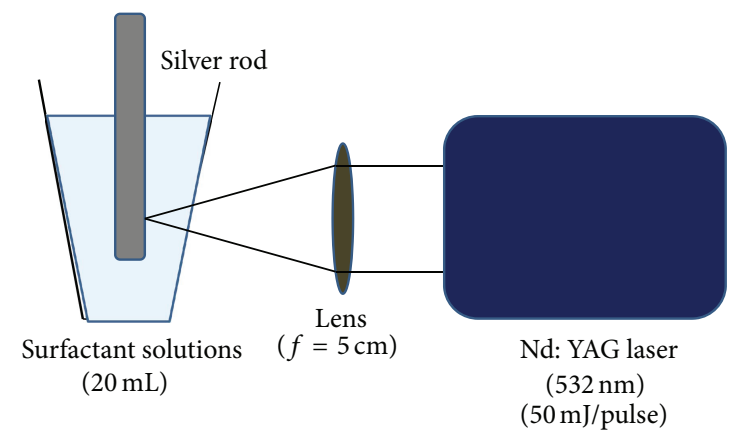

FIGURE 1: Schematic of the experimental setup.

anionic SDS and the cationic CTAB exhibited different stability in protecting Ag colloids during the laser ablation. Later Sobhan et al. [5] investigated the size distribution of gold nanoparticles prepared by femtosecond laser ablation in an aqueous solution of CTAB and found that the mean particle diameter of gold nanoparticles produced in $\mathrm{CTAB}$ was smaller (in the range of 4-6 $\mathrm{nm}$ ) compared to that produced in pure deionized water.

Recently the relationship between the surface plasmon absorption band of metal nanoparticles and its particle size has been thoroughly investigated by Slistan-Grijalva et al. [6], and they concluded that the peak of surface absorption band in spherical silver nanoparticles was proportional to the particle size up to $20 \mathrm{~nm}$. Using this relationship, the particle diameter formed by the laser ablation in water or in an aqueous solution could be easily evaluated without any timeconsuming and troublesome measurements using high-resolution transmission electron spectroscopy. In the present work the influences of the size distributions for the laser ablated nanoparticles on the kinds of surfactants have further been investigated by measuring the spectral shifts for the surface plasmon bands of silver nanoparticles prepared by nanosecond laser ablation in an aqueous solution of several anionic, cationic, and amphoteric surfactants. Furthermore the influence of the dissolved gas in a solution on silver nanoparticle growth using such laser ablation technique was also investigated.

\section{Experimental}

The silver rod (Nirako, 99.99\%, inner diameter of $10 \mathrm{~mm}$ ) immersed in $20 \mathrm{~mL}$ of an aqueous solution containing various kinds of surfactants tabulated in Table 1 was irradiated by a focused $532 \mathrm{~nm}$ second harmonic light of an nanosecond YAG laser (pulse width of $5 \sim 10 \mathrm{~ns}$, repetition rate of $10 \mathrm{~Hz}$ ) with the intensity of $50 \mathrm{~mJ} /$ pulse for each laser pulse. To focus the laser light, a lens with $5 \mathrm{~cm}$ of focal length was set in front of the silver rod as shown in the schematic figure of experimental setup (see Figure 1).

After the 5 min focused laser irradiation to the silver rod immersed in an aqueous solution, the color of the aqueous solution was changed to yellow because of the appearance of the surface plasmon absorption of silver nanoparticles formed by the focused laser irradiation to the silver rod.

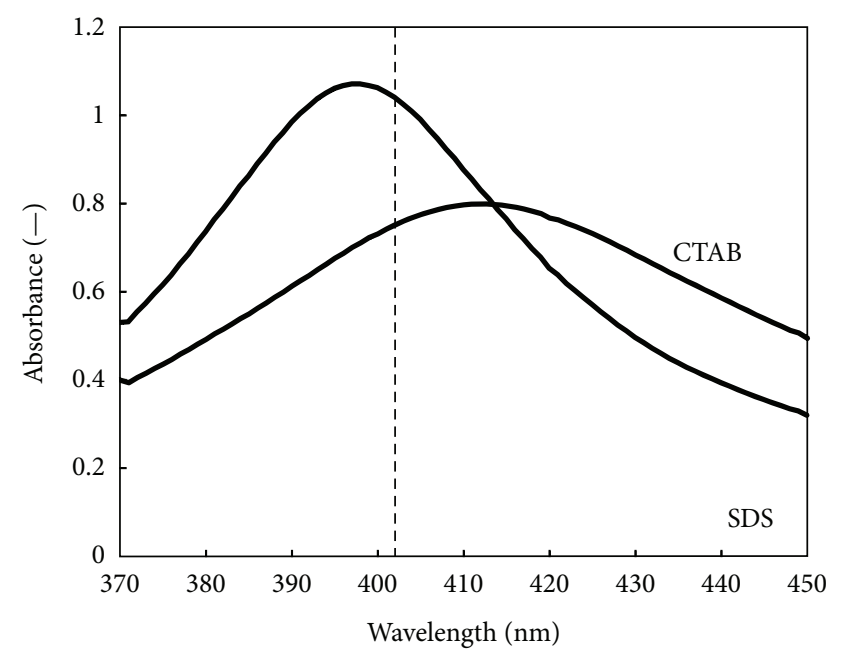

FIGURE 2: Plasmon absorption spectra of silver nanoparticles formed by the focused laser irradiation to silver nanorod in surfactant solution of (a) $10 \mathrm{mM}$ SDS and (b) $1 \mathrm{mM}$ CTAB. The dashed line is the wavelength of the maximum absorption observed by the focused laser irradiation to silver nanorod in pure water.

Finally the yellow solution containing the silver nanoparticles was sampled to a quartz cell, and the spectral shifts for the peak of the surface plasmon bands of silver nanoparticles formed by the focused laser irradiation processes, which are proportional to the mean particle diameter of silver nanoparticles, were measured by the ultraviolet spectrometer (GENESYS 10, Thermo Scientific). To reduce the experimental errors of the spectral shifts for the surface plasmon bands of silver nanoparticles in the present study, these spectral shifts were measured repeatedly more than 5 times in the same experimental conditions, and the averaged spectral shifts were used as the results. Surfactants used in the present work were used without further purification, and deionized water was used to prepare the aqueous solutions for all of the present experimental conditions.

\section{Results and Discussions}

Figure 2 shows the results of the surface plasmon absorption spectra of silver nanoparticles formed by the focused laser irradiation in an aqueous solution containing $10 \mathrm{mM}$ anionic SDS surfactant and $1 \mathrm{mM}$ cationic CTAB surfactant, respectively. The dashed line in Figure 2 indicates the wavelength of the peak for the surface plasmon absorption of silver nanoparticles formed by the focused laser irradiation in a pure water. As shown in Figure 2, the wavelength for the maximum absorption of the surface plasmon band of silver nanoparticles formed by the focused laser irradiation of an aqueous solution containing $10 \mathrm{mM}$ anionic SDS surfactant showed shorter wavelength shift compared to that of silver nanoparticles formed by the focused laser irradiation of pure water $(402 \mathrm{~nm})$. On the other hand, the wavelength for the maximum absorption of the surface plasmon absorption spectra of silver nanoparticles formed by the focused laser irradiation of an aqueous solution containing 
TABLE 1: List of surfactants used in the present work.

\begin{tabular}{|c|c|c|c|}
\hline & Formula & Truncated representation & Company \\
\hline \multicolumn{4}{|l|}{ (a) Anionic surfactant } \\
\hline Sodium dodecyl sulfate & $\mathrm{C}_{12} \mathrm{H}_{25} \mathrm{NaSO}_{4}$ & SDS & Wako \\
\hline Sodium laurate & $\mathrm{C}_{12} \mathrm{H}_{23} \mathrm{NaO}_{2}$ & SL & Tokyo Kasei \\
\hline Sodium 1-dodecanesulfonate & $\mathrm{C}_{12} \mathrm{H}_{25} \mathrm{NaO}_{3} \mathrm{~S}$ & $\mathrm{SD}$ & Tokyo Kasei \\
\hline \multicolumn{4}{|l|}{ (b) Cationic surfactant } \\
\hline Hexadecyltrimethylammonium bromide & $\mathrm{C}_{19} \mathrm{H}_{42} \mathrm{BrN}$ & CTAB & Wako \\
\hline Hexadecyltrimethylammonium chloride & $\mathrm{C}_{19} \mathrm{H}_{42} \mathrm{ClN}$ & CTAC & Tokyo Kasei \\
\hline Trimethylstearylammonium chloride & $\mathrm{C}_{21} \mathrm{H}_{46} \mathrm{ClN}$ & TSAC & Tokyo Kasei \\
\hline 1-Dodecylpyridinium chloride & $\mathrm{C}_{17} \mathrm{H}_{30} \mathrm{ClN}$ & DODAC & Tokyo Kasei \\
\hline \multicolumn{4}{|l|}{ (c) Amphoteric surfactant } \\
\hline Tetradecyldimethyl(3-sulfopropyl)-ammonium hydroxide inner salt & $\mathrm{C}_{19} \mathrm{H}_{41} \mathrm{NO}_{3} \mathrm{~S}$ & TDDSA & Tokyo Kasei \\
\hline Hexadecyldimethyl(3-sulfopropyl)-ammonium hydroxide inner salt & $\mathrm{C}_{21} \mathrm{H}_{45} \mathrm{NO}_{3} \mathrm{~S}$ & HDDSA & Tokyo Kasei \\
\hline
\end{tabular}

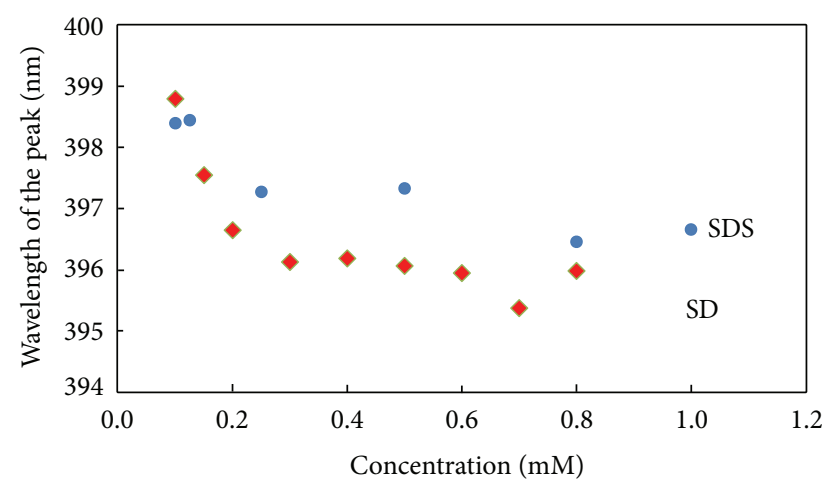

(a)

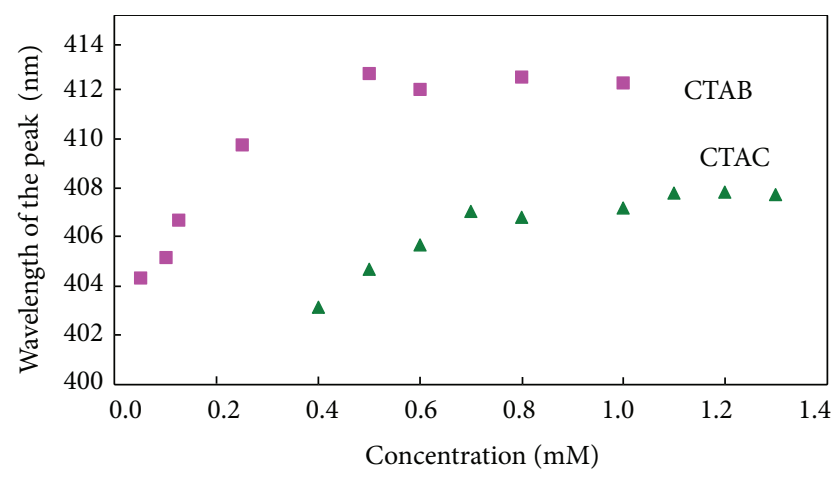

(b)

FIGURE 3: The peak shifts for the plasmon absorption of silver nanoparticles formed by the laser ablation in (a) anionic ( $\bullet$ : SDS, ४: SD) and (b) cationic (๘: CTAB, $\mathbf{\wedge}$ CTAC) surfactants.

$1 \mathrm{mM}$ cationic CTAB surfactant showed longer wavelength shift compared to that of silver nanoparticles formed by the focused laser irradiation of pure water $(402 \mathrm{~nm})$. Based on the theoretical and experimental conclusion by SlistanGrijalva et al. [6] that the maximum absorption wavelength for the surface plasmon absorption band of spherical silver nanoparticles was proportional to the particle size, these experimental results indicated that the particle size of silver nanoparticles formed in $10 \mathrm{mM}$ SDS solution was smaller than that of silver nanoparticles formed in $1 \mathrm{mM} \mathrm{CTAB}$ solution. This result was consistent with the observation of Mafuné et al. [2] and Chen and Yeh [4] but was different from that of Sobhan et al. [5]. The reason why Sobhan et al. observed the decrease of the silver nanoparticle size in focused laser irradiation to solid silver plate was not clear, but it was probably due to the difference of the laser pulse width. To confirm the difference of the peak shift for the plasmon absorption of the silver nanoparticle between anionic and cationic surfactants, spectral shifts of the surface plasmon absorption of silver nanoparticles generated in various kinds of surfactant solutions tabulated in Table 1 were investigated. To confirm the spectral shift of the surface plasmon absorption of the silver nanoparticles, the dependence of the spectral shifts on the concentration of surfactants was firstly investigated. Typical examples of the results were given in Figure 3. As shown in Figure 3, anionic surfactants such as SDS and sodium 1-dodecanesulfonate (denoted by SD hereafter) gave spectral shift toward shorter wavelength. On the other hand, cationic surfactants such as CTAB and hexadecyltrimethylammonium chloride (denoted by CTAC hereafter) gave spectral shift toward longer wavelength. It was also found that at lower surfactant concentrations the spectral shifts became larger with increasing the concentration of surfactants, but at higher surfactant concentration the spectral shifts became constant, although the concentration of the surfactant increased. It is well known that the surfactant starts to form micelles above the concentration of critical micelle concentrations [7]. Thus the concentrations of the free surfactants become constant above the critical micelle concentration. Therefore when the concentrations of the surfactants were above the critical micelle concentrations, the excessed surfactants contribute solely to the component of micelle, and thus the concentration of the free surfactants is always constant above the concentration of critical micelle concentrations. Since only the free surfactants could interact with the precursors of the silver nanoparticles during the laserinduced nanoparticle formation processes and furthermore the surfactants that were the components of micelle could not interact with the precursors of the silver nanoparticles 
during the laser-induced nanoparticle formation processes, it is probably that the saturation of the plasmon spectral shift was only observed at lower surfactant concentrations.

The spectral shifts of the surface plasmon absorption band peaks for the silver nanoparticles formed by the focused laser irradiations in solutions containing the surfactants tabulated in Table 1 relative to the plasmon absorption band of silver nanoparticles formed by the focused laser irradiations in pure water were summarized in Table 2. As shown in Table 2, all of the surface plasmon absorption peaks for the silver nanoparticles formed in solutions containing anionic surfactants were shifted toward shorter wavelength, while all of the surface plasmon absorption peaks for the silver nanoparticles formed in an solution containing cationic surfactants were shifted toward longer wavelength. Mafuné et al. [8] also observed the reduction of gold nanoparticles formed by the focused laser irradiation to the SDS surfactant solutions, and then they proposed a new mechanism called the dynamic formation model. According to their model, a dense cloud of gold atoms (plum) is built over the laser spot of the metal plate after the focused laser irradiation to the solid state. Then the atoms in the cloud are aggregated as fast as the atoms are supplied. This initial rapid aggregation continues until the atoms in close vicinity are depleted almost completely. Thus the metal nanoparticles with relatively uniform size are formed in such laser focusing process. However when the surfactant SDS is added to water, the nanoparticle growth is terminated by coating the particle surface with SDS molecules, and therefore the particle radius formed under the SDS solution was reduced. In the present study the similar phenomena that the size reduction of the silver nanoparticles was observed for the laser ablation processes in anionic surfactant solutions, which is consistent with the dynamic formation model proposed by Mafuné et al. [8]. On the other hand, in the present study the increase of the size in silver nanoparticles was observed in the cationic surfactant solutions. These results were seemingly contradicted to the dynamic formation models proposed by Mafuné et al. [8]. However when we suppose that water also acts as an anionic surfactant and thus interact with the embryonic silver nanoparticles formed by the focused laser irradiation in an solution, then the increase of the particle size due to the cationic surfactant could also be explained using the dynamic formation model because cationic surfactant prevented water to interact with the silver nanoparticles. Thus the interactions between the silver nanoparticles and surfactants were found to be dependent on the charge of surfactants (in other words, the differences between anionic and cationic surfactants), and these differences also affected the silver nanoparticle growth rates during the focused laser irradiation processes toward silver rod immersed in an aqueous solution containing surfactants.

Among surfactants, there are surfactants containing both anionic and cationic functional groups in the same molecule called amphoteric surfactant. In the present study we have also investigated the effect of the silver nanoparticle growth for the amphoteric surfactant as tabulated in Table 1 to understand the interaction between the silver nanoparticles and the amphoteric surfactant by measuring the dependence of the spectral shifts on the concentration of the amphoteric

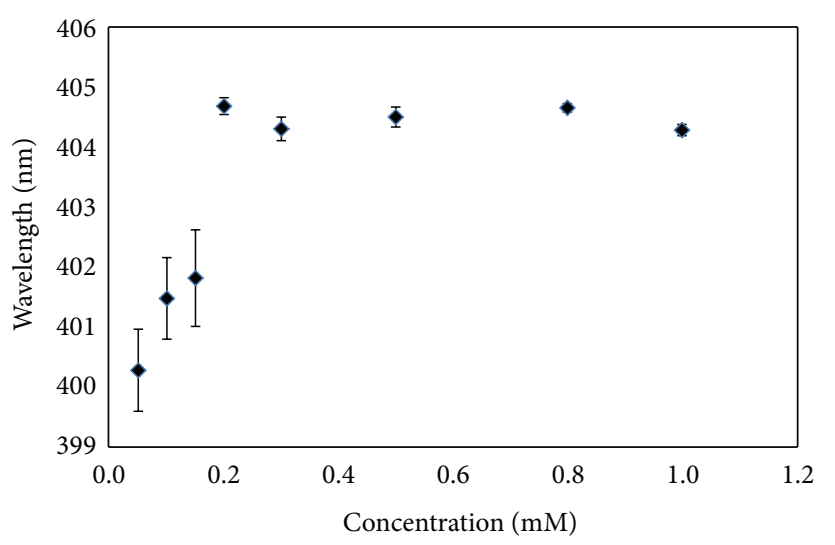

(a)

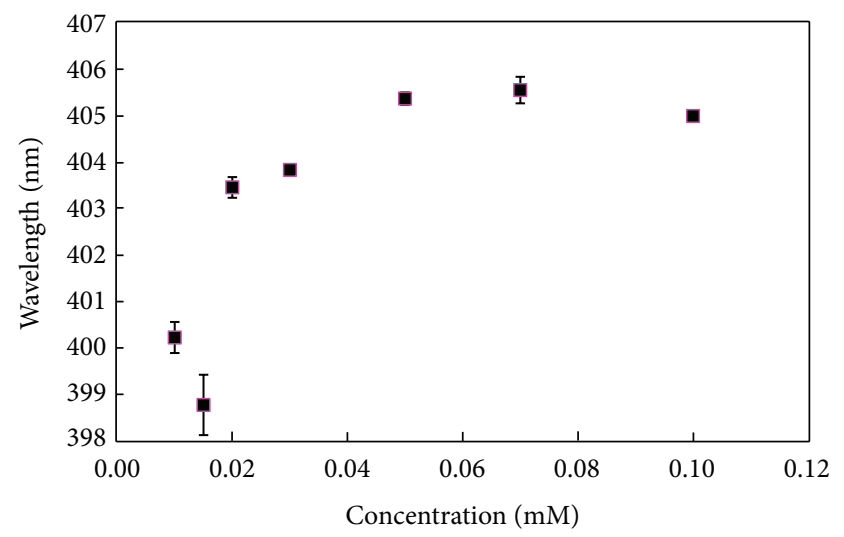

(b)

FIGURE 4: The peak shifts for the plasmon absorption of silver nanoparticles formed by the laser ablation in amphoteric ( TDDSA, $\mathbf{m}:$ HDDSA) surfactants.

surfactants. The results are shown in Figure 4. Interestingly, at lower surfactant concentration the surface plasmon absorption of silver nanoparticle shifted to shorter wavelength compared to that of pure water (i.e., less than $402 \mathrm{~nm}$ ), which corresponds to the behaviors of anionic surfactants such as SDS. But with high surfactant concentration the surface plasmon absorption of silver nanoparticle shifted to longer wavelength, which corresponds to the behaviors of cationic surfactants such as CTAB. Thus the amphoteric surfactant showed both anionic and cationic characters. Since it is also well known that the charge of amphoteric surfactant changes with $\mathrm{pH}$, we have also investigated the influence of the surface plasmon shift of silver nanoparticles formed by the focused laser-irradiation to silver rod immersed in an aqueous solution containing amphoteric surfactants. To prevent the effect of the other ions on the plasmon shift of silver nanoparticles, we have used $\mathrm{HCl}$ and $\mathrm{NaOH}$ to control the $\mathrm{pH}$ of the solution in the present study. The results are shown in Figure 5. As shown in Figure 5, the surface plasmon shift was positive up to $\mathrm{pH}=10$ because of the higher amphoteric surfactant concentrations, but when the $\mathrm{pH}$ becomes from 11 to 12 , the surface plasmon shift suddenly drops to negative. The reason is not clear at this moment, but because of the spectral shift 
TABLE 2: Peak shifts for the surface plasmon absorption band of silver nanoparticles formed by the focused laser irradiation in various kinds of surfactant solutions.

\begin{tabular}{lccccccc}
\hline & \multicolumn{3}{c}{ Anionic surfactant } & \multicolumn{3}{c}{ Cationic surfactant } \\
\hline Sample & SDS & SD & SL & CTAB & CTAC & TSAC & DODAC \\
Wavelength/nm & 397 & 396 & 401 & 412 & 408 & 409 & 406 \\
Shift/nm & -5 & -6 & -1 & +10 & +6 & +7 & +4 \\
\hline
\end{tabular}

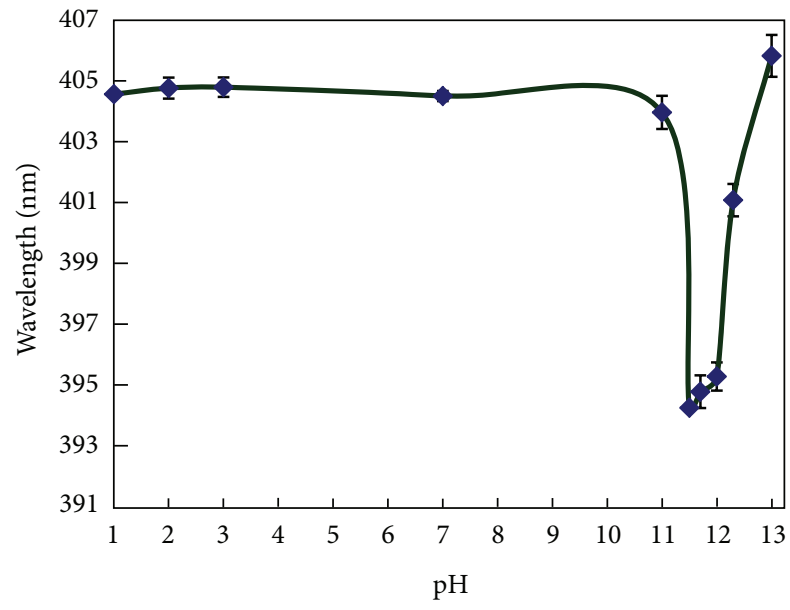

(a)

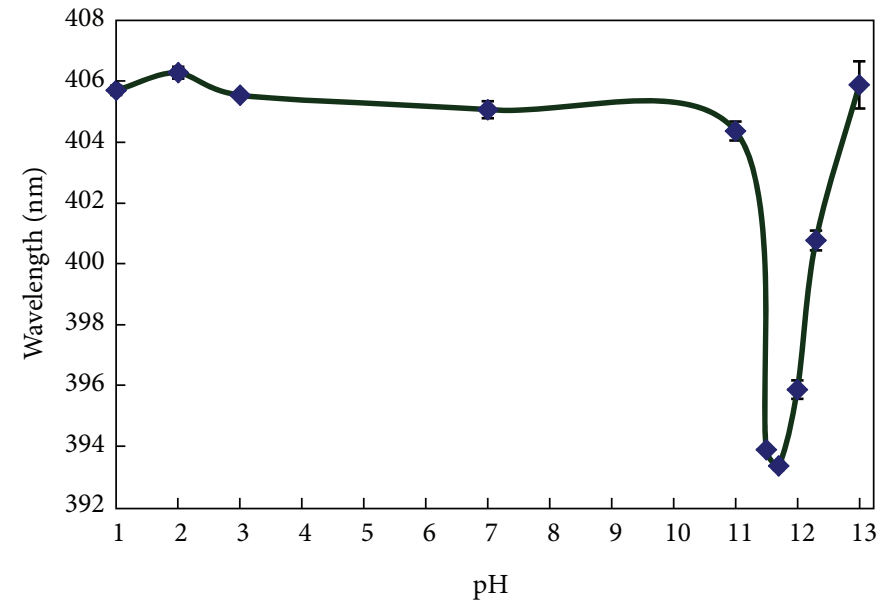

(b)

FIGURE 5: $\mathrm{pH}$ dependence of the peak shifts for the plasmon absorption of silver nanoparticles formed by the laser ablation in amphoteric (a) TDDSA and (b) HDDSA surfactants.

strongly related to the interaction between the silver nanoparticles and the charge of the surfactants, it was probable that the adsorption site of amphoteric surfactants toward silver nanoparticles changed when the $\mathrm{pH}$ was between 11 and 12 . Thus the complicated behaviors of the amphoteric surfactants against the silver nanoparticles growth by the focused laser irradiation to silver rod were found in the present study. Further work should be done to clarify the reasons.

The influences of the surfactants on the silver nanoparticle growth have been investigated so far. However very few works have been carried out for the effect of dissolved gas on the silver nanoparticles growth by the focused laser irradiation to silver rod. Werner et al. [9] recently reported that the efficient size reduction of gold nanoparticle under the supercritical water environment and explained the reason as the interference of bubble and the precursors of gold nanoparticles. However no further studies about the particle growth under the existence of bubble have been carried out so far. In the present study we have also carried out the measurements of the surface plasmon shift of silver nanoparticles formed by the focused laser irradiation to silver rod immersed in an aqueous solution by changing the amount of dissolving the $\mathrm{CO}_{2}$ gas in water, which is known to have high dissolving ability toward water [10]. The result was shown in Figure 6. For comparison the spectrum of the silver nanoparticles in an aqueous solution with dissolved $\mathrm{O}_{2}$ gas is also given in Figure 6. As shown in Figure 6, when the added gas was oxygen, the surface plasmon absorption spectra were tailored toward longer wavelength without any spectral peak shift (see Figure 6(a)). It was consistent with the previous report by the group of Werner et al. [11]. This is probably due to the oxidation of the surface of the silver nanoparticles. On the other hand, when the gas was changed to $\mathrm{CO}_{2}$, the surface plasmon absorption peak was shifted toward longer wavelength (see Figure 6(b)). Since the amount of dissolved $\mathrm{CO}_{2}$ gas in solution can be controlled by changing the gas pressure of $\mathrm{CO}_{2}$ in a reactor, we have investigated the effect of the amount of the $\mathrm{CO}_{2}$ gas dissolved in an aqueous solution on the spectral shift of the plasmon absorption peak wavelength for the silver nanoparticles formed by the focused laserirradiation to silver rod. The result was shown in Figure 7. As shown in Figure 7, the surface plasmon band peak of silver nanoparticles was shifted to longer wavelength by increasing the amount of dissolved $\mathrm{CO}_{2}$ gas. These results indicated that the dissolved gas in an aqueous solution promotes the growth of silver nanoparticles. It is probable that the dissolved gas in a solution became a bubble immediately after the focused laser irradiation, and the bubble promotes the silver nanoparticle growth because of the prevention of the coating as well as the heat loss by water.

\section{Conclusions}

The influence of the particle size of silver nanoparticles formed by the focused laser irradiation to silver rod was investigated by measuring the peak shift of the surface plasmon absorption. It was revealed that anionic surfactants gave shorter wavelength shifts, while cationic surfactants gave longer wavelength shifts. The peak shifts for the silver nanoparticles under the amphoteric surfactant solutions showed 


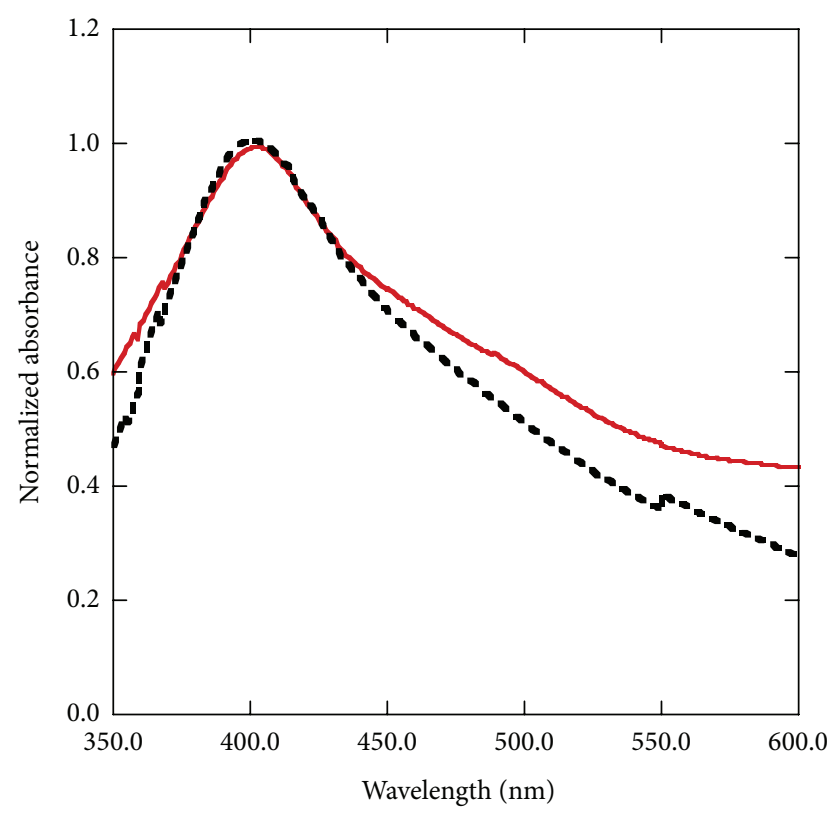

(a)

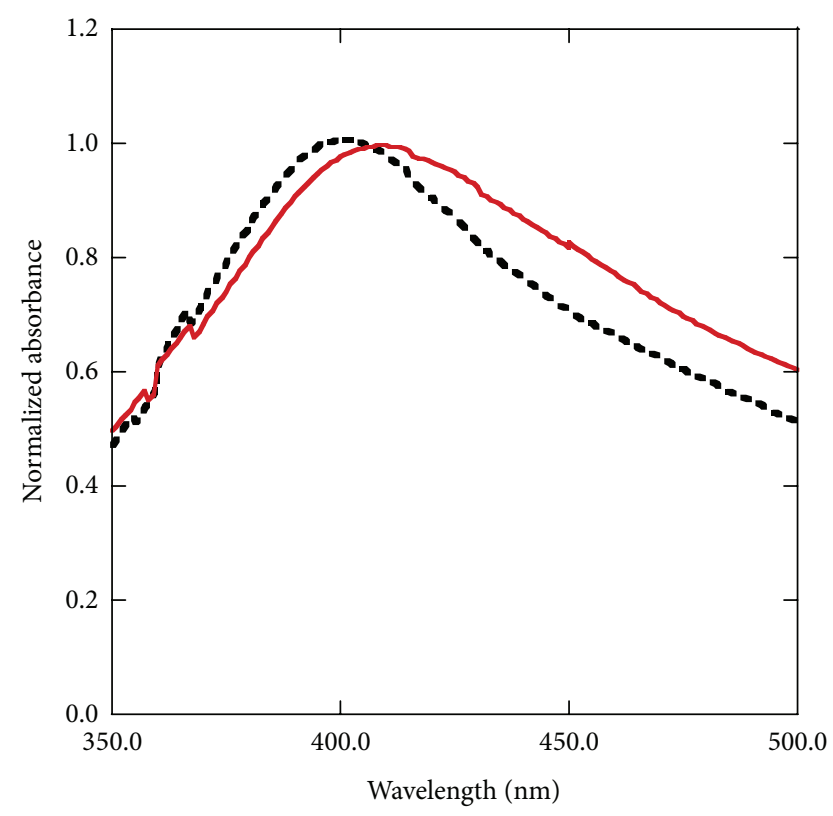

(b)

FIGURE 6: Plasmon absorption spectra of silver nanoparticles formed by the focused laser irradiation to silver nanorod in water with the back pressure of (a) $1 \mathrm{MPa}$ of $\mathrm{O}_{2}$ gas and (b) $12 \mathrm{MPa}$ of $\mathrm{CO}_{2}$. The dashed line is the plasmon absorption spectra of silver nanoparticles formed by the focused laser irradiation to silver nanorod in pure water.

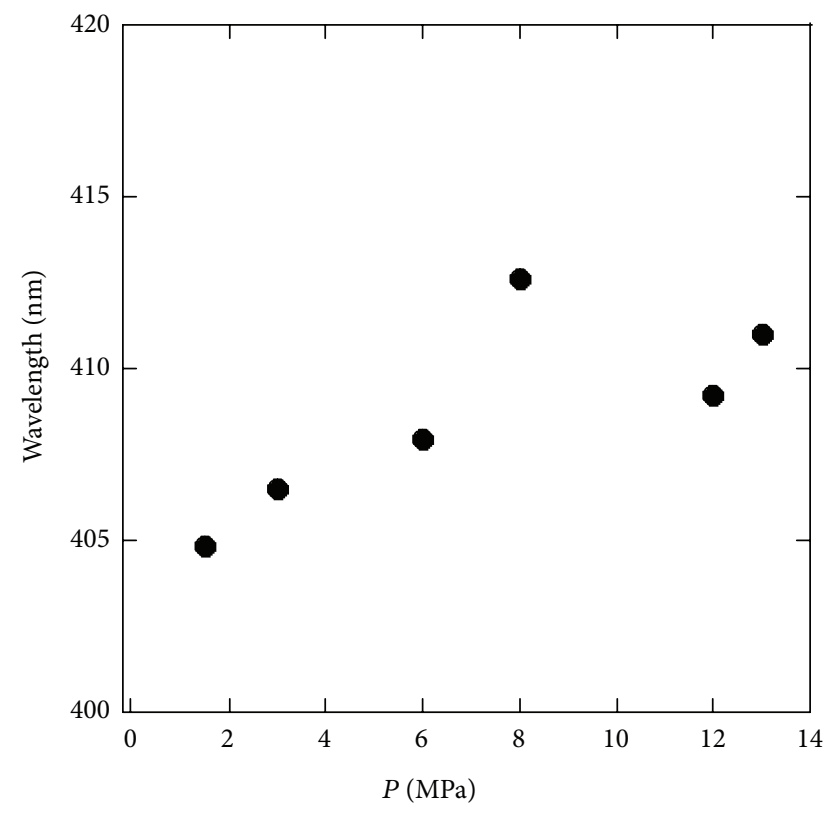

FIGURE 7: The peak shifts for the plasmon absorption of silver nanoparticles formed by the laser ablation in water with the back presssure of 0 to $12 \mathrm{MPa}$ of $\mathrm{CO}_{2}$.

anionic surfactant behavior at lower concentration and cationic surfactant behavior at higher concentration. By changing the $\mathrm{pH}$ of the amphoteric surfactant solution, the abrupt change of the peak shift of the surface plasmon band of silver nanoparticles was observed at $\mathrm{pH}=11-12$.
Finally the effects of the dissolved gas on the peak shift of the surface plasmon band of silver nanoparticles were investigated. It is probable that the dissolved gas in a solution became a bubble immediately after the focused laser irradiation, and the bubble promotes the silver nanoparticle growth.

\section{Acknowledgments}

This work was supported by JSPS KAKENHI Grant number 17760607 and partly by JSPS Adaptable and Seamless Technology Transfer Program through target-drive R\&D.

\section{References}

[1] M. A. El-Sayed, "Some interesting properties of metals confined in time and nanometer space of different shape," Accounts of Chemical Research, vol. 34, no. 4, pp. 257-264, 2001.

[2] F. Mafuné, J. Kohno, Y. Takeda, T. Kondow, and H. Sawabe, “Formation and size control of silver nanoparticles by laser ablation in aqueous solution," Journal of Physical Chemistry B, vol. 104, no. 39, pp. 9111-9117, 2000.

[3] F. Mafune, Y. Kohno, Y. Takeda, and T. Kondow, "Formation of stable platinum nanoparticles by laser ablation in water," The Journal of Physical Chemistry B, vol. 107, no. 18, pp. 4218-4223, 2003.

[4] Y. J. Chen and C. S. Yeh, "Laser ablation method: use of surfactants to form the dispersed Ag nanoparticles," Colloids and Surfaces A, vol. 197, no. 1-3, pp. 133-139, 2002.

[5] M. A. Sobhan, M. J. Withford, and E. M. Goldys, "Enhanced stability of gold colloids produced by femtosecond laser synthesis in aqueous solution of CTAB," Langmuir, vol. 26, no. 5, pp. 3156-3159, 2010. 
[6] A. Slistan-Grijalva, R. Herrera-Urbina, J. F. Rivas-Silva, M. Ávalos-Borja, F. F. Castillón-Barraza, and A. Posada-Amarillas, "Classical theoretical characterization of the surface plasmon absorption band for silver spherical nanoparticles suspended in water and ethylene glycol," Physica E, vol. 27, no. 1-2, pp. 104-112, 2005.

[7] M. J. Rosen and J. T. Kunjappu, Surfactants and Interfacial Phenomena, John Wiley \& Sons, New York, NY, USA, 4th edition, 2012.

[8] F. Mafuné, J. Kohno, Y. Takeda, T. Kondow, and H. Sawabe, "Formation of gold nanoparticles by laser ablation in aqueous solution of surfactant," Journal of Physical Chemistry B, vol. 105, no. 22, pp. 5114-5120, 2001.

[9] D. Werner, T. Ueki, and S. Hashimoto, "Methodological improvement in pulsed laser-induced size reduction of aqueous colloidal gold nanoparticles by applying high pressure," Journal of Physical Chemistry C, vol. 116, no. 9, pp. 5482-5491, 2012.

[10] P. Atkins, Physical Chemistry, Oxford Higher Education, 7th edition, 2001.

[11] D. Werner, S. Hashimoto, T. Tomita, S. Matsuo, and Y. Makita, "Examination of silver nanoparticle fabrication by pulsed-laser ablation of flakes in primary alcohols," Journal of Physical Chemistry C, vol. 112, no. 5, pp. 1321-1329, 2008. 

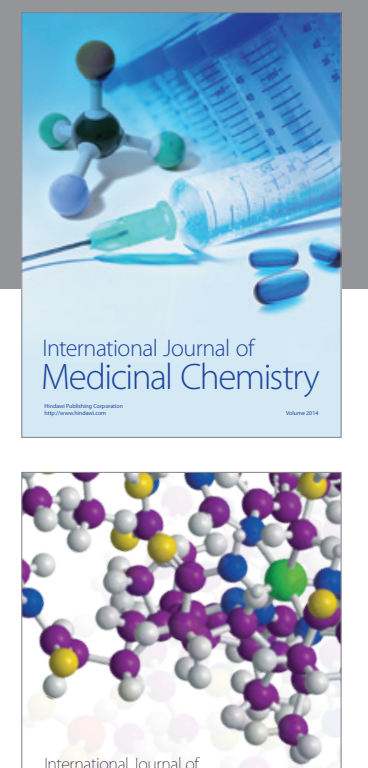

\section{Carbohydrate} Chemistry

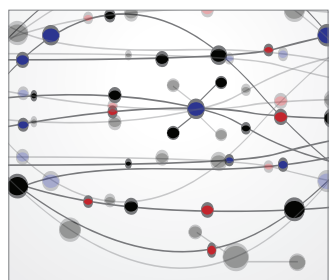

The Scientific World Journal
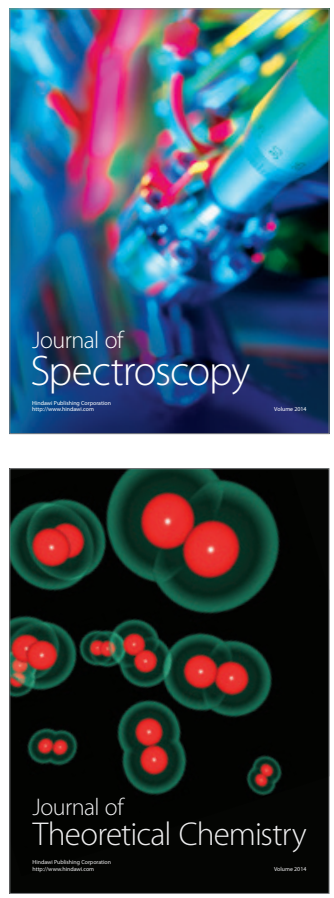
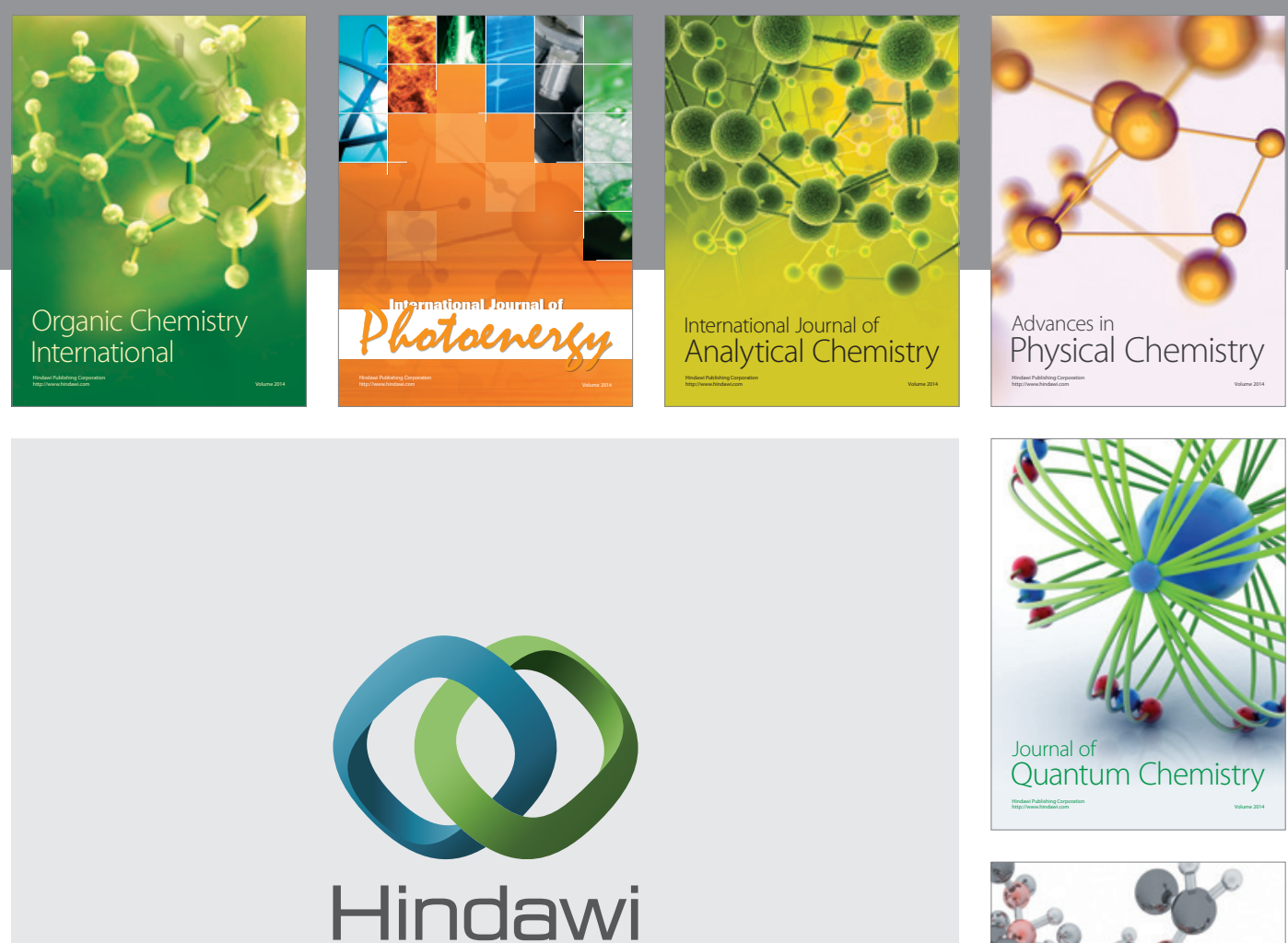

Submit your manuscripts at

http://www.hindawi.com

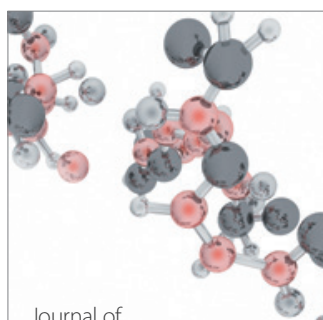

Analytical Methods

in Chemistry

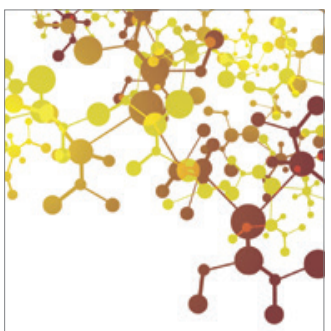

Journal of

Applied Chemistry

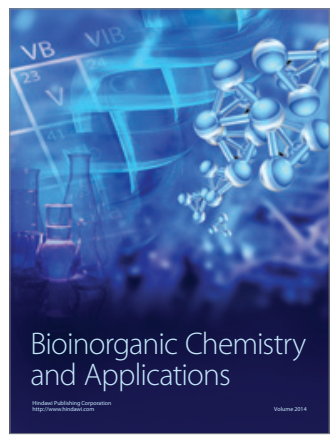

Inorganic Chemistry
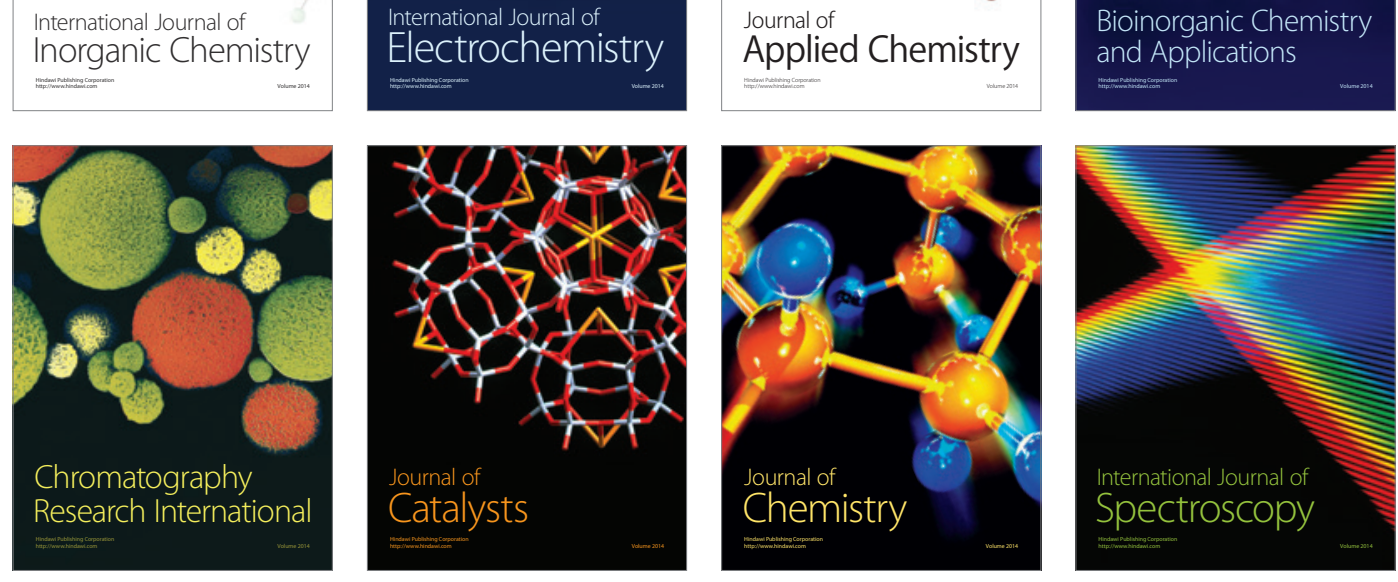